\title{
BP-VE-2
}

\section{Robot transduodenal ampullectomy}

\author{
Yang Won $\mathrm{NAH}^{*}{ }^{* 1}$, Sang Min KONG ${ }^{1}$, Eun Ji LEE' ${ }^{1}$ Sung Jo BANG ${ }^{2}$ \\ 'Department of Surgery, Ulsan University Hospital, Ulsan, Korea \\ ${ }^{2}$ Department of Internal Medicine, Ulsan University Hospital, Ulsan, Korea
}

Introduction: Adenomas arising from the ampulla of Vater (AoV) are premalignant lesions with risk of malignant transformation to carcinoma. Accordingly, many experts advocate resection either endoscopically or surgically. The procedure of choice to resect this rare tumor, however, has still been controversial among endoscopic papillectomy (EP), transduodenal surgical ampullectomy (TDA) and pancreatoduodenectomy (PD). With the introduction of EP recent years, TDA was regarded as a tool for unsuitable lesion for EP or after unsuccessful EP. In addition there might be a role of TDA for preinvasive early stage adenocarcinoma of AoV, substituting PD. Laparoscopic approach replaced open surgery in almost all the abdominal operations such as cholecystectomy, adrenalectomy, gastrectomy and even hepatectomy or pancreatectomy. However, transduodenal ampullectomy requires suturing from all the 24 hour's direction in a small and deep location, making laparoscopic approach almost unfeasible. In contrast, robotic approach could provide almost all the degree of freedom for suturing movement and also provide magnified 3D images, making robot TDA a realistic tool. I'd like to show a video clip for TDA.

Methods: Robot TDA was performed successfully for a $3.7 \mathrm{~cm}$ sized villotubular adenoma in a 53-year-old lady.

Results: The operation took 345 minutes. No blood product was given intraoperatively. Margin-negative resection was confirmed pathologically. The patient was discharged 7 days after the operation with no complication.

Conclusions: Robot transduodenal ampullectomy was feasible and provided the benefit of minimally invasive surgery for otherwise requiring huge open wound for removing a very small specimen. 